\title{
Optimization of transformation of measurements of ship hull blocks
}

\author{
Aleksander Kniat, Ph. D. \\ Gdansk University of Technology
}

\begin{abstract}

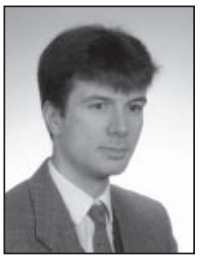

The paper presents an analysis of measurements taken on ship hull blocks to assess their feasibility for the final stage of assembly in a dry dock or on a slipway. The analysis requires first to check if blocks were manufactured within tolerances assumed in design and then to compare if two neighbouring blocks can be joined to each other. As each block is measured in a different coordinate system it is necessary to make transformations and bring results to a common CAD model without loss of accuracy. An algorithm for optimizing the transformation process to obtain better results, is proposed. The optimization is aimed at minimizing the sum of distances between transformed points and corresponding points in a CAD model. Description of the optimization method and example of its application is also presented. The problem of transformation of measurements or coordinate systems is more general as it can be found, apart from shipbuilding, e.g. in civil engineering, cartography, robot control, pattern recognition, medical imaging.
\end{abstract}

Keywords: co-ordinate system transformation; ship hull measurements; ship hull assembly; computer aided design (CAD)

\section{INTRODUCTION}

The dimension control of pre-fabricated hull structural units and blocks is very important in the process of building ship's hull. It is crucial for the shipyard to be sure that units and blocks being brought to a dry dock or slipway have dimensions within assumed tolerances and that they do not require corrections as the time necessary to join them should be as short as possible. Corrections of units and blocks, conducted outside workshop where they have been manufactured, are very time consuming. Moreover it slows down the final hull assembly which is usually a bottleneck in every shipyard production process.

Two comparisons should be done to check dimensions of the units and blocks. It is first necessary to check if the dimensions are within design tolerances. If yes, then two neighbouring blocks should be checked against each other to know where differences occur and of what size they are. This information may influence the way in which the neighbouring units and blocks are joined.

Prefabricated elements are usually measured by means of modern laser-optic instruments. Results of the measurements are presented as a set of coordinates of points in three dimensional space. To verify the dimensions it is necessary to compare location of measured points with a CAD model. Thus the measured points must be brought to the CAD model coordinate system. This requires an isometric transformation of the measurement results to be performed. Usually such transformation is done intuitively by an experienced specialist who checks the dimensions. To avoid human errors and make the process faster an algorithm intended to be implemented as a computer program and hence to solve the problem, is proposed.

\section{MEASUREMENTS - ACCURACY, DIFFICULTIES}

Measurements must be done with proper accuracy. Accuracy is one of the main characteristics of measuring instrument. Only measurements taken with the use of a proper accuracy instrument may be further analyzed. Unfortunately most popular instruments used in land surveys or at construction sites are not precise enough for shipbuilding purposes.

Measurements of ship's hull units and blocks are difficult. There are two main reasons of the difficulties. The first reason is location of measured points within unit or block. Measured points are usually not located at the edges, but inside and they are hidden behind stiffeners, longitudinal or transverse elements. The second reason is position of a given unit or block against its neighbourhood. Prefabricated units and blocks are often stored close to each other as shown in Fig. 2, because of limited space in shipyard. In such cases certain measurement points may be accessible only from one side of a unit or block and the rest from the other side. Taking direct measurements is rather impossible in narrow gaps between units and blocks or in hidden locations therefore additional techniques must be applied. It is very important not to lose 


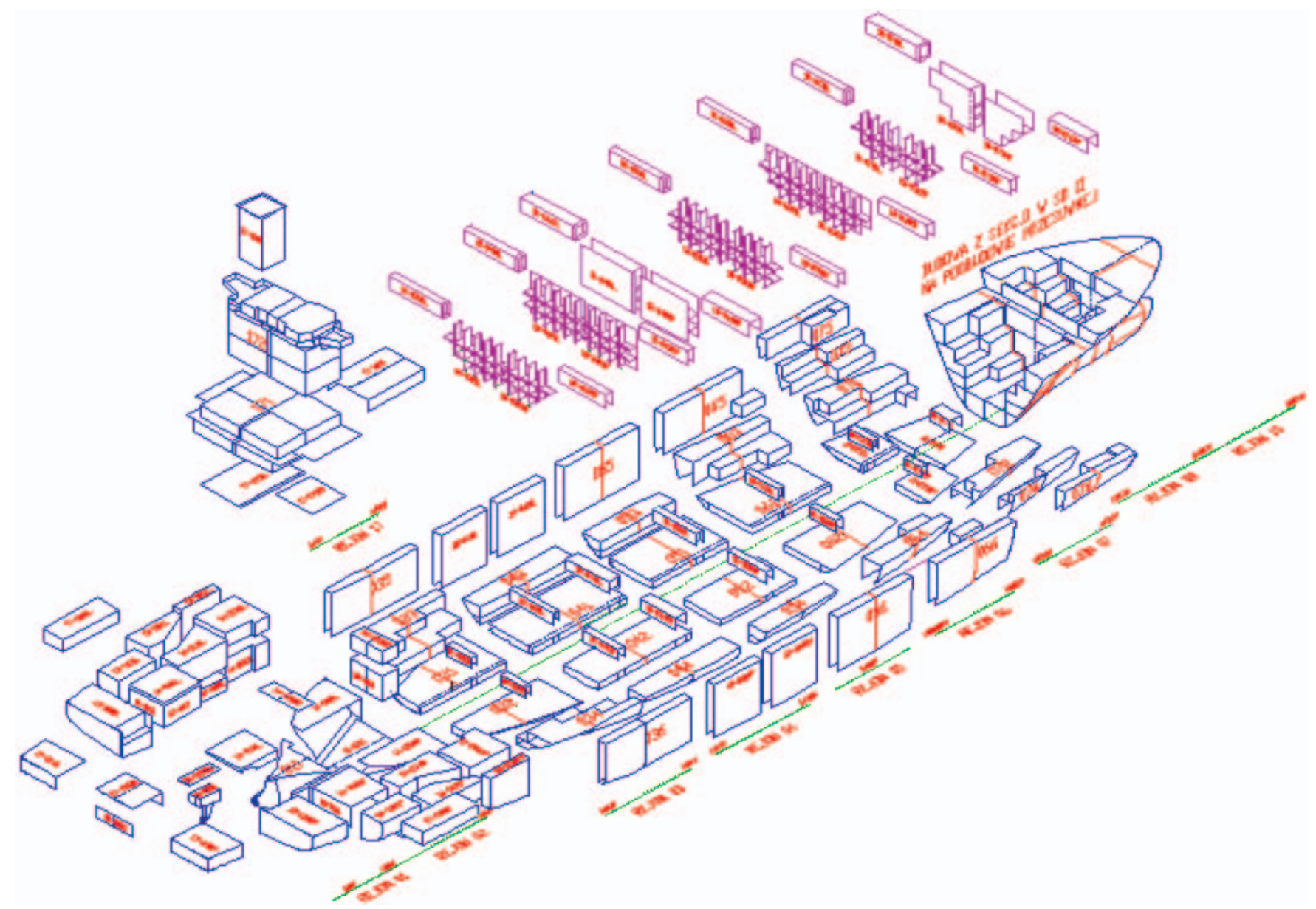

Fig. 1. Units and blocks of ship's hull in an exploded view
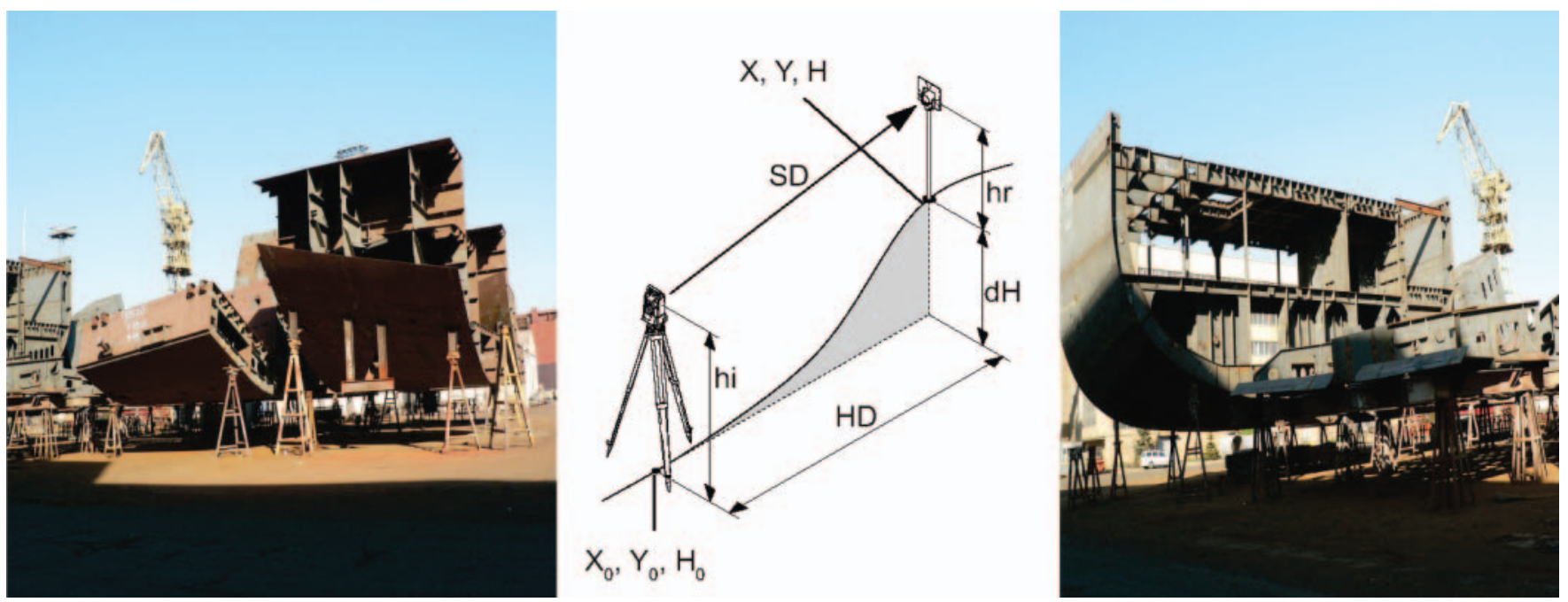

Fig. 2. Prefabricated ship's hull units and blocks in positions in which they are measured

accuracy while applying the techniques because it may lead to incorrect results.

If one unit or block is measured from different positions it is also very important to properly relate both series of measurements to each other. It is achieved either by choosing certain points common for both series of measurements or by establishing a common coordinate system with the use of measuring instrument functions.

There are many different measuring techniques, e.g. with application of visible laser, invisible laser, with reflector, without reflector, automatic search, scanning etc. Each of them has its advantages and disadvantages. However the problem is not discussed in this paper. Attention is focused on transforming results of measurements to assess if prefabricated units and blocks are suitable for further assembly. It is assumed that measurements are taken with a satisfying accuracy.

\section{TRANSFORMATION OF MEASUREMENTS}

Measurements of each unit or block are taken in different coordinate systems. The coordinate system is arranged according to local situation where the measurements are taken. All measured points have corresponding points in the design and in the other unit or block with which it will be 
joined. Therefore the transformation is constrained by the following:

- it must keep distances between measured points within a unit or block,

- it must not lose accuracy.

The purpose of the transformation is to obtain the best fit between measured points and points taken from CAD model or the other unit or block. In other words, the transformation of measurement results is aimed at minimization of the sum of distances between corresponding points.

The problem of transformation of measurements is very similar to the shape recognition or pattern recognition problem. In the shape recognition problem, however, we search for transformation of one set of points to best fit to the other set of points in situation we do not know if the two sets of points correspond to each other. Results of the best transformation show how much similar the two sets of points are. In the case of question we know that points in the two sets correspond to each other and we search for an optimum transformation.

\section{Shape recognition problem}

Shape recognition is a well known problem. In most general form its definition is as follows:

\section{Definition 1}

Given two sets of points $\boldsymbol{A}\left\{\boldsymbol{a}_{1}, \boldsymbol{a}_{2}, \boldsymbol{a}_{3}, \ldots, \boldsymbol{a}_{\boldsymbol{k}}\right\}$ and $\boldsymbol{C}\left\{\boldsymbol{c}_{1}, \boldsymbol{c}_{2}\right.$, $\left.\boldsymbol{c}_{3}, \ldots, \boldsymbol{c}_{\boldsymbol{m}}\right\}$ in d-dimensional space, where $\boldsymbol{k}$ is not necessarily equal to $\boldsymbol{m}$, find the similarity transformation parameters giving the minimum value of the squared distance between corresponding points in these two point sets.

Since the1980s different solutions of the problem were described in many sources e.g. [1, 2, 3, 5, 7, 8, 9, 10].

There are several kinds of the problem depending on:

- known/unknown correspondence between points in the two sets

- equal/unequal number of points in the two sets (phantom points)

- only distance preserving transformation allowed/scaling transformation allowed.

Flick \& Jones [5] give a very general formulation of a d-dimension to d-dimension mapping problem with the possibility of omission, phantom and unknown transformation. Their basic idea is to write down the probability equation for the image based on a given object by all the possible matchings, and then to find the best parameters and the best matching by using the maximum probability principle. However, due to the large number of parameters in the equation and the computational complexity in doing all the possible matchings, a solution is generally intractable unless the number of points is small.

When the correspondences between the point sets are unknown a priori a popular approach to solving the problem is the class of algorithms based on the Iterated Closest Point (ICP) technique introduced by Besl [3] and Zhang [11]. ICP is attractive because of its simplicity and performance. Although the initial estimate does need to be reasonably good, the algorithm converges relatively quickly.

Arun et al. [1] address the ,absolute orientation problem” of finding the least squares solution of a rotation and translation of rigid body transformation. The algorithm [1] sometimes gives a reflection instead of rotation transformation. Umeyama [10] presents a refinement to [1] that always gives a correct rotation matrix and also handles scaling transformation.
Ramos \& Verriest [9] point out that while [10] returns a correct rotation in the degenerate cases where [1] fails, it does so at the expense of larger fitting errors. Ramos \& Verriest [9] propose a method based on a mixed least squares - total least squares solution, assuming noises to be present in both point series to be fitted. The methods presented in $[1,9,10]$ have the disadvantage of requiring both data sets to have the same number of points, and that the point-to-point correspondence is known a priori. On the contrary, Goodrich et al. [6] solve the ,approximate geometric pattern matching problem”, based on the approximate minimizing of the direct Hausdorff distance from the pattern set to the background set, conducted by using rigid body transformation. The point sets can be of different size and the point-to-point correspondence is not assumed.

\section{Definition of measurement transformation problem}

The problem of measurement transformation is a special case of shape recognition problem. In the case in question we assume that the correspondence between the points in the sets is known exactly and the scaling when transforming one set of points into the other, is not allowed. Formal definition of the problem is as follows:

\section{Definition 2}

Given two sets of points $\boldsymbol{A}\left\{\boldsymbol{a}_{1}, \boldsymbol{a}_{2}, \boldsymbol{a}_{3}, \ldots, \boldsymbol{a}_{k}\right\}$ and $\boldsymbol{C}\left\{\boldsymbol{c}_{1}, \boldsymbol{c}_{2}\right.$, $\left.c_{3}, \ldots, c_{k}\right\}$, we search for isometric transformation of points in the set $\boldsymbol{A}$ which produces minimum sum of distances with corresponding points in the set $\boldsymbol{C}$. We assume that each point of the set $\boldsymbol{A}$ has exactly one corresponding point in the set $\boldsymbol{C}$ and each point of the set $\boldsymbol{C}$ has exactly one corresponding point in the set $\boldsymbol{A}$. Points are labelled and their coordinates in 3-dimensional space are known.

For us, isometric transformation is a composition of translations and rotations. We do not allow symmetry (mirror) which is also isometric transformation. Formula (1) defines isometric transformation matrix I which depends on six parameters: $\mathrm{x}, \mathrm{y}, \mathrm{z}$ - coordinates of translation and $\chi, \beta, \alpha$ angles of rotation about $\mathrm{X}, \mathrm{Y}$ and $\mathrm{Z}$ axes.

$$
\begin{gathered}
\text { I(x, } \mathrm{y}, \mathrm{z}, \chi, \beta, \alpha)=\mathbf{T}(\mathrm{x}, \mathrm{y}, \mathrm{z}) \cdot \mathbf{R}(\chi, \beta, \alpha) \\
\text { OPTIMIZATION OF MEASUREMENT } \\
\text { TRANSFORMATION }
\end{gathered}
$$

According to Definition 2 given in the previous paragraph, we must define criterion function for the measurement transformation problem. We assume distance to be the metrics in 3-dimensional space. The square of the distance is also a metrics and it is less complicated for calculation hence the criterion function is presented in the form of the formula (2).

$$
\mathrm{f}=\sum_{\mathrm{i}=1}^{\mathrm{k}}\left(\left\|\mathbf{c}_{\mathbf{i}}-\mathbf{a}_{\mathbf{i}}\right\|_{2}\right)^{2}
$$

where:

$\mathrm{k}$ - number of points in the set $\mathbf{C}$ and the set $\mathbf{A}$

Since we transform $\mathbf{a}_{\mathrm{i}}$ points with isometric transformation matrix $\mathbf{I}(\mathrm{x}, \mathrm{y}, \mathrm{z}, \chi, \beta, \alpha)$ our criterion function depends on six decision variables as presented in formula (3).

$$
\mathrm{f}(\mathrm{x}, \mathrm{y}, \mathrm{z}, \chi, \beta, \alpha)=\sum_{\mathrm{i}=1}^{\mathrm{k}}\left(\left\|\mathbf{c}_{\mathrm{i}}-\mathbf{I}(\mathrm{x}, \mathrm{y}, \mathrm{z}, \chi, \beta, \alpha) \cdot \mathbf{a}_{\mathrm{i}}\right\|_{2}\right)^{2}
$$

By using the criterion function (3) it is possible to calculate an approximate value of the sum of distances between 
corresponding points in the set $\mathbf{C}$ and transformed points in the set $\mathbf{A}$. The aim is to find such values of the decision variables $\mathrm{x}, \mathrm{y}, \mathrm{z}$ and $\chi, \beta, \alpha$, which produce the minimum result of the criterion function (3).

\section{Other possible optimization criteria and constraints}

Measurement results of sections and blocks are transformed and analyzed to assess if sections and blocks can be joined together. Minimum sum of distances between points on seams on both joined sections or blocks is the most obvious criterion but not the only one to be used. While building the ship's hull it is crucial to keep decks planar and adjusted to axes. A measure of how coplanar or how coaxial the sections and blocks are, might also be used as an optimization criterion. Alternatively, it can be used as a constraint, hence only these transformations which yield coplanar and coaxial results within certain tolerance, are accepted.

\section{Optimization method}

There are many methods to minimize function (3). Briefly, they can be divided into two categories: deterministic and probabilistic. Deterministic methods are proven to find a solution when optimized function has certain properties. Not all functions conform to restrictions of deterministic methods.

In our case the criterion function (3) is well defined. To find the minimum for the function (3) we use a deterministic method - the Newton's method described by the formula (4).

$$
\mathbf{x}_{\mathbf{n}+1}=\mathbf{x}_{\mathbf{n}}-\mathbf{H}^{-1} \cdot \mathbf{g}
$$

where:

$\mathbf{x}$ - a vector of decision variables.

This is an iterative method described by Bjorck \& Dahlquist in [4], which requires calculation of the gradient $\mathbf{g}$ and Hessian matrices $\mathbf{H}$ of the function (3). To start, we assume some initial values of the vector of decision variables $\mathbf{x}_{n}$ and calculate next values of the vector of decision variables $\mathbf{x}_{n+1}$. In each step we receive values of decision variables, which produce smaller result of the function (3). The advantage of the Newton's method is a quick convergence and stability.

\section{Point - to - point correspondence}

In case of measurement analysis, exactly one measured point must correspond to exactly one point in a CAD model or located on the other section or block. The one- to- one- point correspondence is a very fundamental assumption. This is so

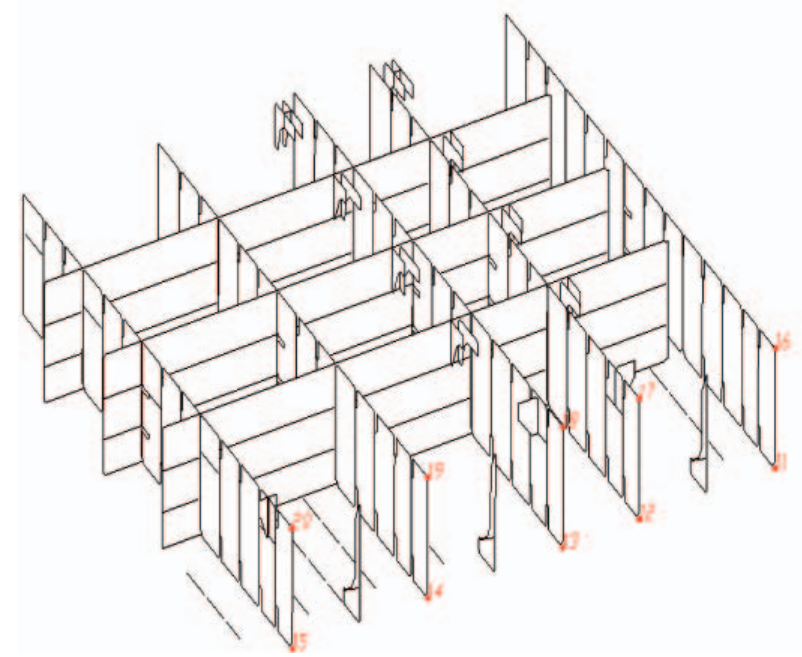

important, because in contrast to the shape recognition problem, it is not the aim to check whether the point sets resemble each other, which is always true, but to precisely state the minor differences between point sets. In case of shape recognition, it is a perfect match if the two patterns of size of about 10 meters have distances between corresponding points of about $30 \mathrm{~mm}$. In case of measurement transformation it is of a great importance if to minimize this distance from $30 \mathrm{~mm}$ to $10 \mathrm{~mm}$, is possible at all.

Labels in point sets allow to control the correspondence between points in the two sets. Assigning labels is time consuming, but prevents from mirror matchings. As the constructions in question are usually symmetric, sometimes about two axes, it is quite easy to lose orientation without a proper labelling. The labelling also prevents from matching sections or blocks which are similar in shape but are not to be joined. Clear labelling system of measured points helps in storing and retrieving measurement results.

\section{Application example results}

The above described method was implemented in a program used for visualization and checking of measurements of ship's hull units and blocks. Measurements of two neighbouring sections of a ship's double bottom were taken as an example. Fig. 3 shows a view of both sections.

Tab.1 presents comparison of measured points and points from CAD model of the portside section, where measurement transformation was done by intuition. Tab. 2 presents the same comparison, but the transformation was optimized by the program.

Tab. 3 presents comparison of measured points and points from CAD model of the starboard section, where measurement transformation was done by intuition. Tab. 4 presents the same comparison, but transformation was optimized by the program.

In both cases the optimized transformation is better. In the case of the portside section the sum of distances is only slightly smaller and the maximum distance between points decreased from $19,36 \mathrm{~mm}$ to $17,50 \mathrm{~mm}$. In the case of the starboard section the difference is more significant. The sum of distances is two times smaller and the maximum distance decreased from $30,05 \mathrm{~mm}$ to $17,21 \mathrm{~mm}$.

\section{DIRECTIONS OF FUTURE RESEARCH}

Further research investigations are expected to be conducted in two main directions: the automatic recognition of measured points and the automatic selection of points to be measured. The

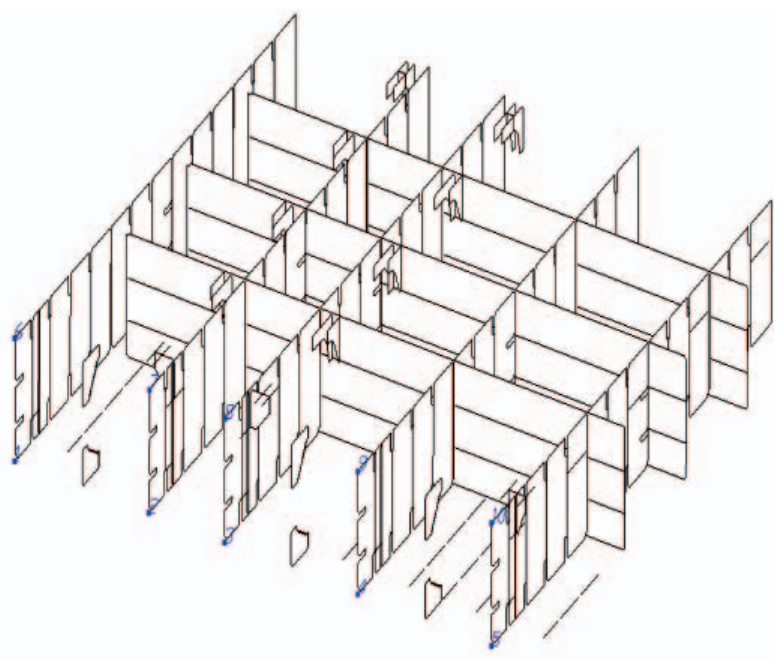

Fig. 3. Neighbouring sections of a ship's hull double bottom with measured points marked with numbers 
Tab. 1. Comparison of measured points and points from CAD model of the portside section - intuitive transformation

\begin{tabular}{|c|c|c|c|c|c|c|c|c|c|c|c|}
\hline \multicolumn{4}{|c|}{ CAD model points } & \multicolumn{3}{c|}{ Transformed measured points } & \multicolumn{4}{c|}{ Comparison } \\
\hline Label & $\mathbf{X}$ & $\mathbf{Y}$ & $\mathbf{Z}$ & Label & $\mathbf{X}$ & $\mathbf{Y}$ & $\mathbf{Z}$ & $\mathbf{d X}$ & $\mathbf{d Y}$ & $\mathbf{d Z}$ & dist \\
\hline $\mathbf{1 0 1}$ & 133400 & 0 & 0 & $\mathbf{0 1}$ & 133413.87 & 0.00 & 0.00 & -13.87 & 0.00 & 0.00 & 13.87 \\
\hline $\mathbf{1 0 2}$ & 130200 & 0 & 0 & $\mathbf{0 2}$ & 130194.79 & 1.10 & 0.18 & 5.21 & -1.10 & -0.18 & 5.32 \\
\hline $\mathbf{1 0 3}$ & 128400 & 0 & 0 & $\mathbf{0 3}$ & 128384.61 & 0.68 & 2.49 & 15.39 & -0.68 & -2.49 & 15.60 \\
\hline $\mathbf{1 0 4}$ & 125200 & 0 & 0 & $\mathbf{0 4}$ & 125202.71 & 0.00 & 3.83 & -2.71 & 0.00 & -3.83 & 4.69 \\
\hline $\mathbf{1 0 5}$ & 122000 & 0 & 0 & $\mathbf{0 5}$ & 122000.00 & 0.00 & 0.00 & 0.00 & 0.00 & 0.00 & 0.00 \\
\hline $\mathbf{1 0 6}$ & 133400 & 0 & 1950 & $\mathbf{0 6}$ & 133409.37 & 15.59 & 1944.57 & -9.37 & -15.59 & 5.43 & 18.98 \\
\hline $\mathbf{1 0 7}$ & 130200 & 0 & 1950 & $\mathbf{0 7}$ & 130192.01 & 0.54 & 1950.39 & 7.99 & -0.54 & -0.39 & 8.02 \\
\hline $\mathbf{1 0 8}$ & 128400 & 0 & 1950 & $\mathbf{0 8}$ & 128380.91 & 0.16 & 1953.21 & 19.09 & -0.16 & -3.21 & $\mathbf{1 9 . 3 6}$ \\
\hline $\mathbf{1 0 9}$ & 125200 & 0 & 1950 & $\mathbf{0 9}$ & 125195.10 & -0.51 & 1950.17 & 4.90 & 0.51 & -0.17 & 4.93 \\
\hline $\mathbf{1 1 0}$ & 122000 & 0 & 1950 & $\mathbf{1 0}$ & 121995.62 & 0.00 & 1951.82 & 4.38 & 0.00 & -1.82 & 4.74 \\
\hline
\end{tabular}

Tab. 2. Comparison of measured points and points from CAD model of the portside section - optimized transformation

\begin{tabular}{|c|c|c|c|c|c|c|c|c|c|c|c|}
\hline \multicolumn{4}{|c|}{ CAD model points } & \multicolumn{3}{c|}{ Transformed measured points } & \multicolumn{4}{c|}{ Comparison } \\
\hline Label & $\mathbf{X}$ & $\mathbf{Y}$ & $\mathbf{Z}$ & Label & $\mathbf{X}$ & $\mathbf{Y}$ & $\mathbf{Z}$ & $\mathbf{d X}$ & $\mathbf{d Y}$ & $\mathbf{d Z}$ & dist \\
\hline $\mathbf{1 0 1}$ & 133400 & 0 & 0 & $\mathbf{0 1}$ & 133417.11 & -3.66 & 0.15 & -17.11 & 3.66 & -0.15 & $\mathbf{1 7 . 5 0}$ \\
\hline $\mathbf{1 0 2}$ & 130200 & 0 & 0 & $\mathbf{0 2}$ & 130198.04 & -0.65 & -0.14 & 1.96 & 0.65 & 0.14 & 2.07 \\
\hline $\mathbf{1 0 3}$ & 128400 & 0 & 0 & $\mathbf{0 3}$ & 128387.86 & 0.00 & 1.90 & 12.14 & 0.00 & -1.90 & 12.29 \\
\hline $\mathbf{1 0 4}$ & 125200 & 0 & 0 & $\mathbf{0 4}$ & 125205.95 & 1.21 & 2.77 & -5.95 & -1.21 & -2.77 & 6.67 \\
\hline $\mathbf{1 0 5}$ & 122000 & 0 & 0 & $\mathbf{0 5}$ & 122003.24 & 3.11 & -1.52 & -3.24 & -3.11 & 1.52 & 4.74 \\
\hline $\mathbf{1 0 6}$ & 133400 & 0 & 1950 & $\mathbf{0 6}$ & 133412.34 & 9.14 & 1944.74 & -12.34 & -9.14 & 5.26 & 16.23 \\
\hline $\mathbf{1 0 7}$ & 130200 & 0 & 1950 & $\mathbf{0 7}$ & 130194.97 & -4.02 & 1950.07 & 5.03 & 4.02 & -0.07 & 6.44 \\
\hline $\mathbf{1 0 8}$ & 128400 & 0 & 1950 & $\mathbf{0 8}$ & 128383.87 & -3.32 & 1952.62 & 16.13 & 3.32 & -2.62 & 16.68 \\
\hline $\mathbf{1 0 9}$ & 125200 & 0 & 1950 & $\mathbf{0 9}$ & 125198.06 & -2.10 & 1949.11 & 1.94 & 2.10 & 0.89 & 3.00 \\
\hline $\mathbf{1 1 0}$ & 122000 & 0 & 1950 & $\mathbf{1 0}$ & 121998.57 & 0.30 & 1950.29 & 1.43 & -0.30 & -0.29 & 1.49 \\
\hline
\end{tabular}

Tab. 3. Comparison of measured points and points from CAD model of the starboard section-intuitive transformation

\begin{tabular}{|c|c|c|c|c|c|c|c|c|c|c|c|c|}
\hline \multicolumn{4}{|c|}{ CAD model points } & \multicolumn{3}{c|}{ Transformed measured points } & \multicolumn{4}{c|}{ Comparison } \\
\hline Label & $\mathbf{X}$ & $\mathbf{Y}$ & $\mathbf{Z}$ & Label & $\mathbf{X}$ & $\mathbf{Y}$ & $\mathbf{Z}$ & $\mathbf{d X}$ & $\mathbf{d Y}$ & $\mathbf{d Z}$ & dist \\
\hline $\mathbf{1 1 1}$ & 133400 & 0 & 0 & $\mathbf{1 1}$ & 133400.00 & 0.00 & 0.00 & 0.00 & 0.00 & 0.00 & 0.00 \\
\hline $\mathbf{1 1 2}$ & 130200 & 0 & 0 & $\mathbf{1 2}$ & 130185.96 & -0.76 & -2.74 & 14.04 & 0.76 & 2.74 & 14.33 \\
\hline $\mathbf{1 1 3}$ & 128400 & 0 & 0 & $\mathbf{1 3}$ & 128375.40 & -1.28 & -0.42 & 24.60 & 1.28 & 0.42 & 24.64 \\
\hline $\mathbf{1 1 4}$ & 125200 & 0 & 0 & $\mathbf{1 4}$ & 125186.42 & -2.56 & -0.45 & 13.58 & 2.56 & 0.45 & 13.83 \\
\hline $\mathbf{1 1 5}$ & 122000 & 0 & 0 & $\mathbf{1 5}$ & 121986.27 & 0.00 & 0.00 & 13.73 & 0.00 & 0.00 & 13.73 \\
\hline $\mathbf{1 1 6}$ & 133400 & 0 & 1950 & $\mathbf{1 6}$ & 133401.79 & 0.00 & 1948.05 & -1.79 & 0.00 & 1.95 & 2.65 \\
\hline $\mathbf{1 1 7}$ & 130200 & 0 & 1950 & $\mathbf{1 7}$ & 130178.92 & 2.05 & 1945.82 & 21.08 & -2.05 & 4.18 & 21.58 \\
\hline $\mathbf{1 1 8}$ & 128400 & 0 & 1950 & $\mathbf{1 8}$ & 128370.33 & 3.71 & 1947.05 & 29.67 & -3.71 & 2.95 & $\mathbf{3 0 . 0 5}$ \\
\hline $\mathbf{1 1 9}$ & 125200 & 0 & 1950 & $\mathbf{1 9}$ & 125180.79 & 0.43 & 1949.68 & 19.21 & -0.43 & 0.32 & 19.21 \\
\hline $\mathbf{1 2 0}$ & 122000 & 0 & 1950 & $\mathbf{2 0}$ & 121980.13 & 3.76 & 1950.81 & 19.87 & -3.76 & -0.81 & 20.24 \\
\hline
\end{tabular}

Tab. 4. Comparison of measured points and points from CAD model of the starboard section-optimized transformation

\begin{tabular}{|c|c|c|c|c|c|c|c|c|c|c|c|}
\hline \multicolumn{4}{|c|}{ CAD model points } & \multicolumn{3}{c|}{ Transformed measured points } & \multicolumn{4}{c|}{ Comparison } \\
\hline Label & $\mathbf{X}$ & $\mathbf{Y}$ & $\mathbf{Z}$ & Label & $\mathbf{X}$ & $\mathbf{Y}$ & $\mathbf{Z}$ & $\mathbf{d X}$ & $\mathbf{d Y}$ & $\mathbf{d Z}$ & dist \\
\hline $\mathbf{1 1 1}$ & 133400 & 0 & 0 & $\mathbf{1 1}$ & 133415.46 & -1.34 & 1.56 & -15.46 & 1.34 & -1.56 & 15.60 \\
\hline $\mathbf{1 1 2}$ & 130200 & 0 & 0 & $\mathbf{1 2}$ & 130201.42 & -0.35 & -1.38 & -1.42 & 0.35 & 1.38 & 2.01 \\
\hline $\mathbf{1 1 3}$ & 128400 & 0 & 0 & $\mathbf{1 3}$ & 128390.86 & 0.32 & 0.83 & 9.14 & -0.32 & -0.83 & 9.19 \\
\hline $\mathbf{1 1 4}$ & 125200 & 0 & 0 & $\mathbf{1 4}$ & 125201.88 & 1.84 & 0.60 & -1.88 & -1.84 & -0.60 & 2.70 \\
\hline $\mathbf{1 1 5}$ & 122000 & 0 & 0 & $\mathbf{1 5}$ & 122001.72 & -0.47 & 0.86 & -1.72 & 0.47 & -0.86 & 1.98 \\
\hline $\mathbf{1 1 6}$ & 133400 & 0 & 1950 & $\mathbf{1 6}$ & 133417.13 & 1.56 & 1949.62 & -17.13 & -1.56 & 0.38 & $\mathbf{1 7 . 2 1}$ \\
\hline $\mathbf{1 1 7}$ & 130200 & 0 & 1950 & $\mathbf{1 7}$ & 130194.26 & -0.24 & 1947.19 & 5.74 & 0.24 & 2.81 & 6.39 \\
\hline $\mathbf{1 1 8}$ & 128400 & 0 & 1950 & $\mathbf{1 8}$ & 128385.67 & -1.77 & 1948.31 & 14.33 & 1.77 & 1.69 & 14.54 \\
\hline $\mathbf{1 1 9}$ & 125200 & 0 & 1950 & $\mathbf{1 9}$ & 125196.13 & 1.77 & 1950.73 & 3.87 & -1.77 & -0.73 & 4.32 \\
\hline $\mathbf{1 2 0}$ & 122000 & 0 & 1950 & $\mathbf{2 0}$ & 121995.47 & -1.32 & 1951.67 & 4.53 & 1.32 & -1.67 & 5.01 \\
\hline
\end{tabular}


points to be measured are now clearly indicated in measurement instructions which are a part of the workshop design. The points are chosen by designers and production engineers who design techniques of the assembly process.

As the ship hull sections and blocks are manufactured it seems possible to use some new marking method to make the points of interest for measurements distinguishable, e.g. paint of a special kind, magnetic or radio location etc. This way, the time-consuming pointing of each measured point by means of a mirror or a pole, could be omitted.

The automatic selection of measured points requires to have knowledge about construction. In order to choose appropriate points this knowledge should be built into the program which makes decisions instead of a human being. It is difficult but might be possible by applying knowledge engineering and artificial intelligence. The other solution could be obtained by using the scanning of entire section or block. The comparing of entire elements may eliminate choice of points to be measured. However the precise scanning of large elements is a problem. Moreover, sections and blocks in a shipyard are usually stored close to each other or close to other constructions like scaffolding, cranes etc. In such conditions separating scanned element from surroundings is a serious task.

\section{CONCLUSIONS}

- Optimization of measurement transformation of ship blocks is very important for the assessment if blocks were manufactured within assumed tolerances and if they can be assembled in a dry dock or on a slipway. The presented method was implemented to the computer program for visualisation and assessment of measurements of ship blocks. Transformation of measurements is done while reading results of measurements in 3-dimensional model of a ship hull. Since the iterative optimization method converges quickly, in about 5 iterations usually, the results are immediate.

The method has the following advantages:

- $\quad$ easy for implementing (derivatives of only trigonometric functions and polynomials)

- quick convergence, immediate results - it requires only a few (about 5) iterations to find a minimum,

- it eliminates analysis of how to align measurement results with a CAD model,

- it eliminates human errors when transforming measurements results into a CAD model.

- The only disadvantage of the method is that it requires exact correspondence between measurement points and CAD model points.

- This method is also assumed to be applied to a possible future research. The transformation of measurements is necessary and not dependent on a way the measured points are selected. From the user point of view, the application of the method which automatically transforms measurements to get the best fit with CAD model, saves time and makes results clear to be assessed.

\section{BIBLIGRAPHY}

1. Arun, K.S. et al.: Least-squares fitting of two point sets. IEEE Transactions on Pattern Analysis and Machine Intelligence, Vol. PAMI-9, No. 5, 1987

2. Baird, H. S.: Model-based image matching using location. MIT Press, Cambridge, MA, 1985

3. Besl, P.J., \& McKay, N.: A method for registration of 3-D shapes. IEEE Transactions on Pattern Analysis and Machine Intelligence, Vol. 14 (2), 1992
4. Bjorck, A., \& Dahlquist, G.: Numerical methods. Prentice-Hall, New Jersey, 1974

5. Flick, T. E., \& Jones, L. K.: A combinational approach for classification of patterns with missing information and random orientation. IEEE Transactions On Pattern Analysis And Machine Intelligence, Vol. PAMI-8, 1986

6. Goodrich, M.T. et al.: Approximate geometric pattern matching under rigid motion. IEEE Transactions on Pattern Analysis and Machine Intelligence, Vol. PAMI-21, No. 4, 1999

7. Griffin, P. M., \& Alexopoulos, C.: Point pattern matching using centroid bounding. IEEE Transactions on Systems, Man, and Cybernetics, Vol. SMC-19, 1989

8. Lavine D., Lambird B. A., \& Kanal L. N.: Recognition of spatial point patterns. Pattern Recognition, Vol. 16, 1983

9. Ramos, J.A., \& Verriest, E.I.: Total least squares fitting of two point sets in $m-D$. Proceedings of the 36 th Conference on Decision and Control, 1997

10.Umeyama, S.: Least-squares estimation of transformation parameters between two point patterns. IEEE Transactions on Pattern Analysis and Machine Intelligence, Vol. PAMI-13, No. 4, (1991)

11.Zhang, Z.: On local matching of free-form curves. Proceedings of the British Machine Vision Conferences, 1992

\section{ADDITIONAL READING}

12.Brockett, R.W.: Singular values and least squares matching. Proceedings of the 36th IEEE Conference on Decision and Control, Vol. 2, 1997

13.Fitzgibbon, A.W. Robust registration of $2 \mathrm{D}$ and $3 \mathrm{D}$ point sets. Image and Vision Computing, Vol. 21, No. 13-14 Elsevier Science B.V., 2003 http://www.robots.ox.ac.uk/ awf/lmicp

14.Gaojin, W., Dengmin, G.Z., Shihong, X., Zhaoqi, W: Total least squares fitting of point sets in $m-D$, Computer Graphics International, 2005

15.Gronwall, C., Anderson, P., Gustafsson, F.: Least squares fitting of articulated objects. Proceedings of IEEE Proceedings - Computer Vision and Pattern Recognition (CVPR'05), Computer Society, 2005

16.Gunnarsson, K.T., Prinz, F.B.: CAD model-based localization of parts in manufacturing. Computer, Vol. 20, No. 8, 1987

17.Kluth, V.S., Kunkel, G.W., Rauhala, U.A.: Global least squares matching. Proceedings of Geoscience and Remote Sensing Symposium (IGARSS '92), Vol. 2, 1992

18.Manninen, M., Kaisto, I.: 3D measurement and analysis of a ship block. Practice report. A.M.S. Ltd, Oulu/Finland, 1996, http://www.leica-geosystems.com/media/new/product solution/ U1-298-0EN AMS.pdf

19. Morgera, S.D., Cheong, P.L.C.: Rigid body constrained noisy point pattern matching. IEEE Transactions on Image Processing, Vol. 4, No. 5, 1995

20.O'leary, P., Harker, M., Zsombor-Murray, P.: Direct and least square fitting of coupled geometric objects for metric vision. IEE Proceedings - Vision, Image and Signal Processing, Vol. 152, No. 6, 2005

21.Tarnowski, W.: Fundamentals of technical design (in Polish). WNT, Warsaw, 1997

22.Yang, M.C.K., \& Lee, J.S.: Object identification from multiple images based on point matching under a general transformation. IEEE Transactions on Pattern Analysis and Machine Intelligence, Vol. 16, No. 7, 1994.

\section{CONTACT WITH THE AUTHOR}

Aleksander Kniat, Ph. D.

Faculty of Ocean Engineering and Ship Technology

Gdansk University of Technology Narutowicza 11/12

80-952 Gdansk, POLAND

e-mail: olek@pg.gda.pl 